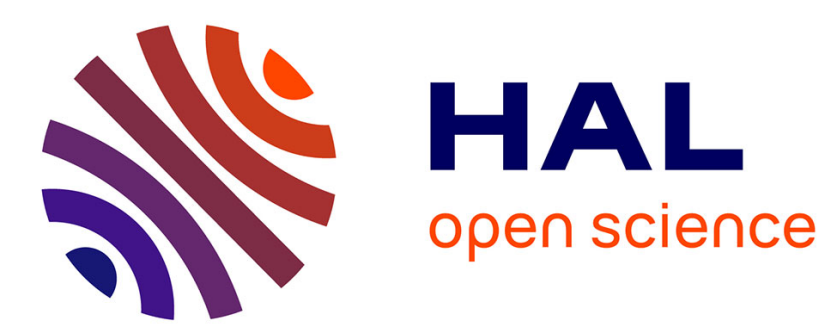

\title{
Detection of microwave radiation using thin film superconducting loops containing weak links
}

\author{
M. Nisenoff
}

\section{To cite this version:}

M. Nisenoff. Detection of microwave radiation using thin film superconducting loops containing weak links. Revue de Physique Appliquée, 1974, 9 (1), pp.65-68. 10.1051/rphysap:019740090106500 jpa00243777

\section{HAL Id: jpa-00243777 https://hal.science/jpa-00243777}

Submitted on 1 Jan 1974

HAL is a multi-disciplinary open access archive for the deposit and dissemination of scientific research documents, whether they are published or not. The documents may come from teaching and research institutions in France or abroad, or from public or private research centers.
L'archive ouverte pluridisciplinaire HAL, est destinée au dépôt et à la diffusion de documents scientifiques de niveau recherche, publiés ou non, émanant des établissements d'enseignement et de recherche français ou étrangers, des laboratoires publics ou privés. 


\title{
DETECTION OF MICROWAVE RADIATION USING THIN FILM SUPERCONDUCTING LOOPS CONTAINING WEAK LINKS
}

\author{
M. NISENOFF
}

\author{
US Naval Research Laboratory, Washington, DC 20375, USA
}

\begin{abstract}
Résumé. - Des SQUID à film supraconducteur excités à $30 \mathrm{MHz}$ ont été utilisés pour la détection de rayonnements électromagnétiques dans une bande de fréquence s'étendant de $1 \mathrm{kHz}$ jusqu'à $10 \mathrm{GHz}$. En excitant ces dispositifs en hyperfréquences il est possible de détecter un rayonnement de longueur d'onde millimétrique ou sub-millimétrique. La sensibilité escomptée pour un rayonnement de $100 \mathrm{GHz}$ est de l'ordre de $10^{-17} \mathrm{~W} / v^{\prime} \overline{\mathrm{Hz}}$.
\end{abstract}

Abstract. - Thin film superconducting SQUIDs biased at $30 \mathrm{MHz}$ have been used for the detection of electromagnetic radiation in the frequency range from about $1 \mathrm{kHz}$ up to at least $10 \mathrm{GHz}$. With the improved performance obtained for microwave-excited devices, detection of $\mathrm{mm}$ and sub-mm radiation should be possible. Calculated sensitivity for a microwave excited SQUID to radiation at $100 \mathrm{GHz}$ is about $10^{-17} \mathrm{~W} / \sqrt{\mathrm{Hz}}$.

A superconducting loop containing a weak-link structure [1], [2], [3] - an rf-biased SQUID - can be used as a detector of radiation through-out the frequency spectrum from very low frequency through the far-infrared. In this paper preliminary results will be presented on the operation of this type of device as a radiation detector for frequencies up to the microwave region and a discussion of the use of these devices in the millimeter and sub-millimeter region will be presented.

If the rf-biased SQUID is inductively coupled to a resonant structure (for example, a lumped parameter LC circuit at radio frequencies (1-300 $\mathrm{MHz})$ ) the detected voltage across the resonant structure will have the form [4], [5]

$V=I_{\mathrm{p}} Z \sin \omega_{\mathrm{p}} t+\frac{\omega_{\mathrm{p}} \varphi_{\mathrm{p}} M Q}{L} \sum_{n=1}^{\infty} \cos \left(\frac{2 \pi n}{\varphi_{0}} \varphi(t)\right)$ where $I_{p}$ is the magnitude of a constant amplitude rf current at the frequency $\omega_{\mathrm{p}}$ imposed on the tank circuit at its resonant frequency, $\varphi_{\mathrm{p}}$ is the amplitude of the magnetic flux induced at frequency $\omega_{\mathrm{p}}$ at the site of the SQUID due to the current in the tank circuit, $Q$ is the quality factor of the resonant circuit, $Z$ is the impedance of the loaded tank circuit, $M$ is the mutual inductance between the coil and the device, $L$ is the inductance of the SQUID, $\varphi_{0}$ is the flux quantum and $\varphi(t)$ is an arbitrary time varying flux that the SQUID experiences which is the sum of $\varphi_{\mathrm{p}} \sin \omega_{\mathrm{p}} t$ and other imposed fields.

When an rf-biased SQUID is used as a low frequency magnetometer, it is exposed to a pump frequency flux $\varphi_{\mathrm{p}} \sin \omega_{\mathrm{p}} t$ and to a low frequency magnetic flux $\varphi_{\mathrm{dc}}$. Under these conditions, the response of the device will have the form

$$
V=\left[I_{\mathrm{p}} Z+\frac{\omega_{\mathrm{p}} \varphi_{\mathrm{p}} M Q}{L} J_{1}\left(\frac{2 \pi \varphi_{\mathrm{p}}}{\varphi_{0}}\right) \sin \frac{2 \pi \varphi_{\mathrm{dc}}}{\varphi_{0}}\right] \sin \omega_{\mathrm{p}} t
$$

where only the fundamental component at frequency $\omega_{\mathrm{p}}$ is shown. The behavior of these devices have been extensively studied in the frequency range from 1 to $300 \mathrm{MHz}$ and the form of eq. (2) has been experimentally verified.

However, if the SQUID is to be used to detect radiation at some signal frequency $\omega_{\text {sig }}$, then the time-varying magnetic flux has the form

$$
\varphi(t)=\varphi_{\mathrm{p}} \sin \omega_{\mathrm{p}} t+\varphi_{\mathrm{sig}} \sin \omega_{\mathrm{sig}} t
$$

and eq. (1) becomes

$$
V=I_{\mathrm{p}} Z \sin \omega_{\mathrm{p}} t+\frac{\omega_{\mathrm{p}} \varphi_{\mathrm{p}} M Q}{L} \sum_{n=1}^{\infty} \cos \frac{2 \pi n}{\varphi_{0}}\left[\varphi_{\mathrm{p}} \sin \omega_{\mathrm{p}} t+\varphi_{\mathrm{sig}} \sin \omega_{\mathrm{sig}} t\right] .
$$


The character of the detected voltage $V$ will depend on the parameters of the detection system, viz., its center frequency and the post detection bandwidth. For example, if the detection system is tuned to the difference frequency $\left(\omega_{\mathrm{p}}-\omega_{\mathrm{sig}}\right)$, the voltage across the resonant circuit will have the form

$$
V=\frac{\omega_{\mathrm{p}} \varphi_{\mathrm{p}} M Q}{L} J_{1}\left(\frac{2 \pi \varphi_{\mathrm{sig}}}{\varphi_{0}}\right) J_{1}\left(\frac{2 \pi \varphi_{\mathrm{p}}}{\varphi_{0}}\right) \sin \left(\omega_{\mathrm{p}}-\omega_{\mathrm{sig}}\right) t
$$

In this context, the pump frequency plays the role of the local oscillator power in the more familiar heterodyne detection scheme. This mode of operation of SQUID has been demonstrated earlier by Zimmerman [6] using $30 \mathrm{MHz}$ as the difference frequency.

If the detection system is centered at $\omega_{\mathrm{p}}$, then the voltage across the resonant structure will have the form [7]

$$
\begin{aligned}
& V=\left[I_{\mathrm{p}} Z+\frac{\omega_{\mathrm{p}} \varphi_{\mathrm{p}} M Q}{L} J_{1}\left(\frac{2 \pi \varphi_{\mathrm{p}}}{\varphi_{0}}\right) J_{0}\left(\frac{2 \pi \varphi_{\mathrm{sig}}}{\varphi_{0}}\right)\right] \sin \omega_{\mathrm{p}} t+ \\
& \quad+\frac{\omega_{\mathrm{p}} \varphi_{\mathrm{p}} M Q}{L} J_{1}\left(\frac{2 \pi \varphi_{\mathrm{p}}}{\varphi_{0}}\right) J_{1}\left(\frac{2 \pi \varphi_{\mathrm{sig}}}{\varphi_{0}}\right)\left\{\sin \left(\omega_{\mathrm{p}}+\omega_{\mathrm{sig}}\right) t-\sin \left(\omega_{\mathrm{p}}-\omega_{\mathrm{sig}}\right) t\right\} \\
&+ \\
&+\frac{\omega_{\mathrm{p}} \varphi_{\mathrm{p}} M Q}{L} J_{1}\left(\frac{2 \pi \varphi_{\mathrm{p}}}{\varphi_{0}}\right) J_{2}\left(\frac{2 \pi \varphi_{\mathrm{sig}}}{\varphi_{0}}\right)\left\{\sin \left(\omega_{\mathrm{p}}+2 \omega_{\mathrm{sig}}\right) t+\sin \left(\omega_{\mathrm{p}}-2 \omega_{\mathrm{sig}}\right) t\right\} \\
&+\frac{\omega_{\mathrm{p}} \varphi_{\mathrm{p}} M Q}{L} J_{1}\left(\frac{2 \pi \varphi_{\mathrm{p}}}{\varphi_{0}}\right) J_{3}\left(\frac{2 \pi \varphi_{\mathrm{sig}}}{\varphi_{0}}\right)\left\{\sin \left(\omega_{\mathrm{p}}+3 \omega_{\mathrm{sig}}\right) t-\sin \left(\omega_{\mathrm{p}}-3 \omega_{\mathrm{sig}}\right) t\right\} \\
&+\cdots .
\end{aligned}
$$

If the post detection bandwidth $f_{\mathrm{BW}}$ is less than $\omega_{\mathrm{sig}} / 2 \pi$ then only the component at frequency $\omega_{\mathrm{p}}$ will pass through the amplifier and be envelope detected by the diode detector at the output of the detection system. Thus, the voltage across the amplifier will have the form

$$
V=\left[I_{\mathrm{p}} Z+\frac{\omega_{\mathrm{p}} \varphi_{\mathrm{p}} M Q}{L} J_{1}\left(\frac{2 \pi \varphi_{\mathrm{p}}}{\varphi_{0}}\right) J_{0}\left(\frac{2 \pi \varphi_{\mathrm{sig}}}{\varphi_{0}}\right)\right] \sin \omega_{\mathrm{p}} t .
$$

If the bandwidth of the detection system $f_{\mathrm{BW}}$ is large compared to $\omega_{\mathrm{sig}} / 2 \pi$ then all of the terms in eq. (6) will contribute to the detected signal. Rearranging terms yield the following equation

$$
V=I_{\mathrm{p}} Z \sin \omega_{\mathrm{p}} t+\frac{\omega_{\mathrm{p}} \varphi_{\mathrm{p}} M Q}{L} J_{1}\left(\frac{2 \pi \varphi_{\mathrm{p}}}{\varphi_{0}}\right) \sin \left(\omega_{\mathrm{p}} t+\frac{2 \pi \varphi_{\mathrm{sig}}}{\varphi_{0}} \sin \omega_{\mathrm{sig}} t\right) .
$$

Expanding the term in $\cos (a+b)$ and collecting factors which vary as $\sin \left(\omega_{\mathrm{p}} t\right)$ one obtains,

$$
V=\left[I_{\mathrm{p}} Z+\frac{\omega_{\mathrm{p}} \varphi_{\mathrm{p}} M Q}{L} J_{1}\left(\frac{2 \pi \varphi_{\mathrm{p}}}{\varphi_{0}}\right) \cos \left(\frac{2 \pi \varphi_{\mathrm{sig}}}{\varphi_{0}} \sin \omega_{\mathrm{sig}} t\right)\right] \sin \omega_{\mathrm{p}} t .
$$

Thus the signal varies periodically as the Bessel function of the amplitude of the pump (just as in the case of the low frequency magnetometer) and sinusoidally as low frequency flux. The period of the low frequency response of the device will depend on both the amplitude and the frequency of the low frequency signal. The restriction placed on bandwidth of the detection system takes the form

$$
\frac{2 \pi}{\varphi_{0}} \varphi_{\mathrm{sig}} \omega_{\mathrm{sig}} \leqslant f_{\mathrm{BW}}, \quad(2 \pi)^{2}\left(\frac{\varphi_{\mathrm{sig}}}{\varphi_{0}}\right) f_{\mathrm{sig}} \leqslant f_{\mathrm{BW}} .
$$

These two modes of detection have been verified using a pump frequency of $30 \mathrm{MHz}$ and a signal frequency of $1 \mathrm{kHz}$. The bandwidth of the detection system was varied by changing the capacitance in an RC bandpass filter located after the diode detector. For a bandwidth of $10 \mathrm{kHz}$ the output of the detection system is shown schematically for several values of the amplitude $\varphi_{\text {sig }}$ (see Fig. 1). The top curve is the signal and the lower traces show the output signal for various amplitudes of the signal. These signals are typical of the signal seen from a SQUID magnetometer when the low frequency applied field has the amplitude shown [1]-[5]. From the values of the current in the coil which produced the various curves, the current corresponding to a single flux quantum $\varphi_{0}$ can be deduced.

If the bandwidth of the detection system was reduced to a value small compared to $\omega_{\text {sig }}$ (additional capacitance added to the output terminals of the detection system), then the envelope of the detected 


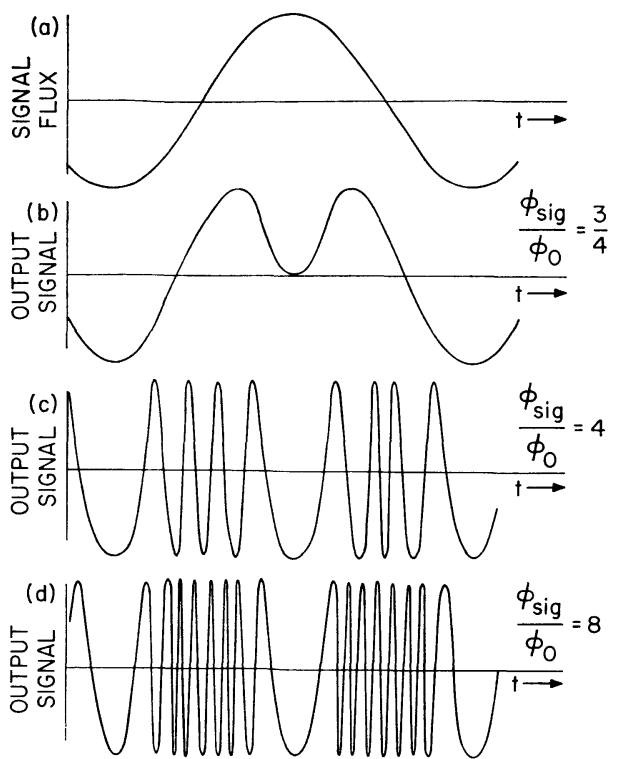

FIG. 1. - Schematic representation of the output signal obtained from a SQUID detection system due to an incident signal at frequency $\omega_{\mathrm{sig}} / 2 \pi$ where $\omega_{\mathrm{sig}} / 2 \pi$ is much smaller than the bandwidth of the detection system. The time variation of the signal, $\varphi(t)=\varphi_{\text {sig }} \sin \omega_{\text {sig }} t$, is shown in trace $(a)$ and the output signals from the detection system for several values of $\varphi_{\text {sig, }}$, the amplitude of the signal, are shown in traces $(b),(c)$ and $(d)$. The shape of the output signals will depend on the ambient dc magnetic flux, and, therefore, an arbitrary value of dc flux, different for each trace, was chosen in the preparation of these traces.

output varied as the zero order Bessel function as shown in figure 2. In this figure, the abscissa was calibrated by the procedure mentioned in the paragraph above and the ordinate was normalized for

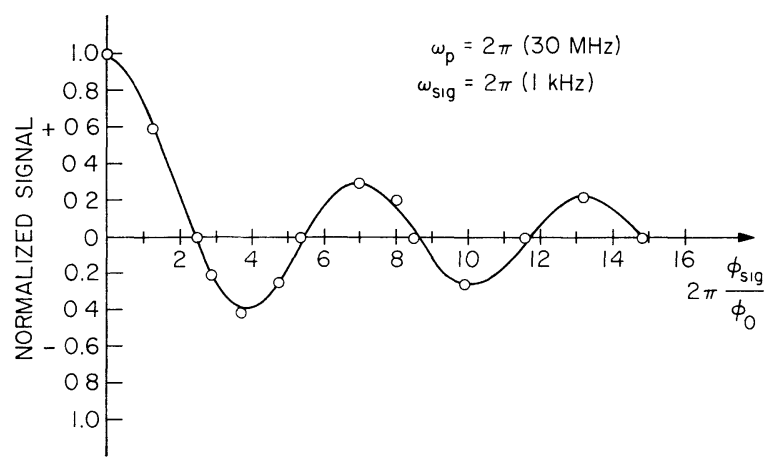

FIG. 2. - Response of SQUID to amplitude of signal at frequency $\omega_{\mathrm{sig}} / 2 \pi$ when $\omega_{\mathrm{sig}} / 2 \pi$ is greater than post-detection band-width of detection system. Data taken for signal frequency of $1 \mathrm{kHz}$ and post detection bandwidth of $100 \mathrm{~Hz}$. The scale for the abscissa is absolute and was determined as described in the text. The signal amplitude was normalized for $\varphi_{\text {sig }}=0$. The solid line is a plot of $J_{0}\left(\frac{2 \pi}{\varphi_{0}} \varphi_{\mathrm{sig}}\right)$.

$\varphi_{\text {sig }}=0$. The solid curve in the figure is the zero order Bessel function with the argument ( $\left.2 \pi \varphi_{\text {sig }} / \varphi_{0}\right)$ The fit of the data to the predicted dependence on $\varphi_{\text {sig }}$ is quite good.

The same type of Bessel dependence was obtained for all values of $\omega_{\text {sig }}$ from about $100 \mathrm{~Hz}$ to $10 \mathrm{GHz}$, provided that the signal frequency was not a harmonic or subharmonic of the pump frequency. This is not an inherent feature of this mode of detection but was probably due to the fact that the input of the detection system was non-linear and any signal « leaking » into the input of the amplifier would have generated multiples and sub-multiples of the signal frequency. If these frequencies were within the bandpass of the detection system, a spurious signal would appear at the output and distort the nature of the detected signal. Appropriate filters should keep the signal frequency out of the input stage of the detection system.

For most frequencies the calibration of the coil could not be determined experimentally and thus the scale of the abscissa cannot be established absolutely but has to be normalized.

This mode of detection using the SQUID is, in many ways, similar to the zero voltage mode of detection of a voltage bias point contact such as used by Richards, Shapiro and their respective co-workers. This mode is signal frequency insensitive and thus responds to all incident signals. The only serious disadvantage of this mode of detection compared to the " difference frequency " mode of detection is that $J_{0}(x)$ has zero slope for small values of the argument while the $J_{1}(x)$ dependence obtained in the difference frequency mode of detection is linear for small values of the argument.

Recently, thin film SQUIDs have been studied in a waveguide system at microwave frequencies, $10 \mathrm{GHz}$ [8], [9]. The SQUIDs, which previously have been measured at $30 \mathrm{MHz}$, were placed in a region of high microwave magnetic field in a $\mathrm{TE}_{102}$ rectangular cavity with the microwave magnetic field along the axis of the cylindrical SQUID. The cavity containing the SQUID was placed in one arm of a microwave bridge which formed a part of a conventional Electron Spin Resonance spectrometer. The general features of the impedance of the cavity were very similar to that observed at radio frequencies, that is, with increasing incident power, once the circulating current induced in the SQUID exceeded the critical current of the weak link, the microwave impedance of the cavity exhibited steps uniformly spaced in (incident power) $)^{1 / 2}$ and the risers of these steps could be modulated by a low frequency magnetic flux applied to the SQUID with a periodicity of the flux quantum $\varphi_{0}$.

The most significant features of the microwave response of the SQUIDs are :

1) the magnitude of the signal corresponds to the change in the reflected power of $10^{-10} \mathrm{~W}$ (compared to a signal of the order of $10^{-12} \mathrm{~W}$ for $30 \mathrm{MHz}$ excitation), and

2) the field modulated signal for $10 \mathrm{GHz}$ excitation 
was of the order of $10^{4}: 1$ for a $10 \mathrm{~Hz}$ postdetection bandpass (compared to about $10^{3}: 1$ signal-tonoise ratio obtained at $30 \mathrm{MHz}$ for a $1 \mathrm{~Hz}$ bandpass) with noise taken as peak to peak.

Thus, microwave excitation has greatly improved the characteristics of the signal obtained for the SQUID as compared to the response at radio frequencies. In fact, the improvement may even be larger as the response at the lower signal frequency has been optimized while with microwave frequency excitation, a careful systematic study of coupling between the devices and the waveguide cavity has not as yet been performed and a careful evaluation of the various detection systems has not been done to select the best low noise amplifier. These studies are presently in process.

With the improved performance obtained with microwave excitation, an estimate can be made of the minimum detectable signal that a SQUID radiation sensor should see at $100 \mathrm{GHz}$. The minimum detectable change in magnetic energy that can be detected is $(\Delta \varphi)^{2} / 2 L$ and the power corresponding to this is

$$
P_{\min }=\left(\frac{(\Delta \varphi)}{2 L}\right)^{2}=\omega_{\text {sig }} \frac{\left[\left(\frac{\Delta \varphi}{\varphi_{0}}\right) \varphi_{0}\right]^{2}}{2 L} .
$$

Assuming that the inductance of the device is $10^{-10} \mathrm{H}$, the low frequency value and that $\Delta \varphi / \varphi_{0} \approx 10^{-4}$, then $P_{\min } \sim 2 \times 10^{-17} \mathrm{~W} / \sqrt{\mathrm{Hz}}$. This value is very encouraging for detectors in the frequency range from $100 \mathrm{GHz}$ and up. Of course, this sensitivity must be experimentally verified.

Summary. - A superconducting loop containing a single weak link structure has potential as a detector of electromagnetic radiation in the $\mathrm{mm}$ and sub-mm frequency range. For the suggested mode of operation the calculated sensitivity for such a device is $2 \times 10^{-17} \mathrm{~W} / \sqrt{\mathrm{Hz}}$ at $100 \mathrm{GHz}$, the detector would have a broad band response to all incident radiation, the device can be readily coupled to a wave guide structure and, in the case of light pipe waveguide structure, it might be possible to couple the SQUID to the dielectric lightpipe by fabricating the device directly on the lightpipe itself. The disadvantage of this suggested mode of operation is that the device cannot be operated in a frequency selective manner and that the power dependence follows a zeroth order Bessel function and thus for small values of the incident magnetic flux the response varies as $1-x^{2} / 4$ compared to a linear dependence on magnetic flux for a first order Bessel dependence.

\section{References}

[1] Silver, A. H. and Zimmerman, J. E., Phys. Rev. 157 (1967) 317.

[2] Zimmerman, J. E., Cryogenics 12 (1972) 19.

[3] Gifford, R. P., Webb, R. A. and Wheatley, J. C., J. Low. Temp. Phys. 6 (1972) 533.

[4] Mercereau, J. E., Revue Phys. Appl. 5 (1970) 13.

[5] Nisenoff, M., Revue Phys. Appl. 5 (1970) 21.
[6] Zimmerman, J. E., J. Appl. Phys. 41 (1970) 1589.

[7] Crimes, C. C. and Shapiro, S., Phys. Rev. 169 (1968) 397.

[8] Rachford, F. J., Huang, C. Y., Wolf, S. and Nisenoff, M., Bull. Amer. Phys. Soc. 18 (1973) 706.

[9] Rachford, F. J., Huang, C. Y., Wolf, S. and Nisenoff, M., To be published. 